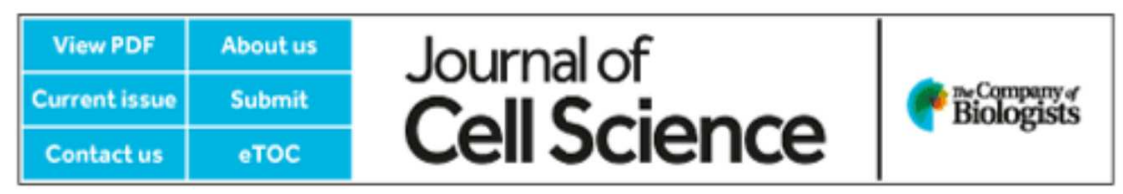

J Cell Sci. 2012 Nov 15; 125(22): 5288-5301.

PMCID: PMC3561852

doi: $10.1242 / j$ jcs. 101030

\title{
Upon Wnt stimulation, Rac1 activation requires Rac1 and Vav2 binding to p120-catenin
}

\author{
Gabriela Valls, ${ }^{1,{ }^{*}}$ Montserrat Codina, ${ }^{1,2,{ }^{*}}$ Rachel K. Miller, ${ }^{3}$ Beatriz Del Valle-Pérez, ${ }^{1}$ Meritxell Vinyoles, ${ }^{1}$ Carme Caelles, ${ }^{3}$ \\ Pierre D. McCrea, ${ }^{4}$ Antonio García de Herreros,${ }^{2,5, \ddagger}$ and Mireia Duñach ${ }^{1, \neq}$ \\ ${ }^{1}$ Departament de Bioquímica i Biologia Molecular, CEB, Facultat de Medicina, Universitat Autònoma de Barcelona, E-08193 Bellaterra, Spain \\ ${ }^{2}$ Programa de Recerca en Càncer, IMIM-Hospital del Mar, E-08003 Barcelona, Spain \\ ${ }^{3}$ Institute for Research in Biomedicine (IRB Barcelona) and Department of Biochemistry and Molecular Biology (Pharmacy), Universitat de Barcelona, \\ E-08028 Barcelona, Spain \\ ${ }^{4}$ Department of Biochemistry and Molecular Biology, University of Texas M.D Anderson Cancer Center, Houston, TX 77030, USA \\ ${ }^{5}$ Departament de Ciències Experimentals i de la Salut, Universitat Pompeu Fabra, E-08003 Barcelona, Spain \\ * These authors contributed equally to this work \\ ${ }^{\ddagger}$ Authors for correspondence (Email: agarcia@imim.es; Email: mireia.dunach@uab.es)
}

Accepted 2012 Jul 16

Copyright @ 2012. Published by The Company of Biologists Ltd

This article has been cited by other articles in PMC.

A role for Rac1 GTPase in canonical Wnt signaling has recently been demonstrated, showing that it is required for $\beta$-catenin translocation to the nucleus. In this study, we investigated the mechanism of Rac 1 stimulation by Wnt. Upregulation of Rac 1 activity by Wnt3a temporally correlated with enhanced p120-catenin binding to Rac1 and Vav2. Vav2 and Rac1 association with p120-catenin was modulated by phosphorylation of this protein, which was stimulated upon serine/threonine phosphorylation by CK1 and inhibited by tyrosine phosphorylation by Src or Fyn. Acting on these two post-translational modifications, Wnt3a induced the release of p120-catenin from E-cadherin, enabled the interaction of p120-catenin with Vav2 and Rac1, and facilitated Rac1 activation by Vav2. Given that p120-catenin depletion disrupts gastrulation in Xenopus, we analyzed p120-catenin mutants for their ability to rescue this phenotype. In contrast to the wild-type protein or other controls, p120-catenin point mutants that were deficient in the release from E-cadherin or in Vav2 or Rac1 binding failed to rescue p120-catenin depletion. Collectively, these results indicate that binding of p120-catenin to Vav2 and Rac1 is required for the activation of this GTPase upon Wnt signaling.

Key words: Wnt signaling, p120-catenin, Rac1, Vav2

Canonical Wnt signaling is essential for correct embryo development, and is involved in the control of cell growth and differentiation of most mammalian cells (Klaus and Birchmeier, 2008; Reya and Clevers, 2005). The downstream response of this pathway is the activation of the $\beta$-catenin/Tcf- 4 transcriptional complex that leads to the expression of a wide variety of target genes (MacDonald et al., 2009; Vlad et al., 2008). The activity of this complex is dependent on the stabilization of $\beta$-catenin: in nonstimulated cells, $\beta$-catenin not bound to cadherins is rapidly degraded through the association with a destruction complex that includes the tumor suppressor adenomatous polyposis gene (APC), Axin, and the Thr/Ser protein kinases CK1 $\alpha$ and glycogen synthase kinase $3 \beta$ (GSK3 $\beta$ ). $\beta$-catenin phosphorylation by GSK3 $\beta$ recruits $\beta \operatorname{TrCP}$ ubiquitin ligase, leading to its proteasomal degradation (Liu et al., 2002). Ligands of the canonical Wnt pathway, such as Wnt3a, through a signaling pathway involving Dvl2 and LRP5/6 phosphorylation, disrupt the destruction complex and inhibit GSK3 $\beta$ activity, stabilizing $\beta$-catenin (Klaus and Birchmeier, 2008; MacDonald et al., 2009). Nuclear localization of 
$\beta$-catenin has also been shown to be dependent on Rac1 activity (Wu et al., 2008), a member of the Rho family of small GTPases that cycle between inactive GDP-bound and active GTP-bound forms (Etienne-Manneville and Hall, 2002; Parri and Chiarugi, 2010). Activation of Rac1 induces the phosphorylation of $\beta$-catenin at Ser191 and Ser605 mediated by JNK2 kinase; these two modifications are required for $\beta$-catenin accumulation in the nucleus (Wu et al., 2008). Moreover, another Rac1-dependent kinase, the p21-activated kinase (PAK), directly phosphorylates $\beta$-catenin at Ser675, contributing to its stabilization (Zhu et al., 2012).

p120-catenin is a pleiotropic protein involved in E-cadherin stabilization. Apart from binding to E-cadherin, p120-catenin associates with other proteins, such as Kaiso transcription factor (Daniel and Reynolds, 1999), RhoA GTPase (Yanagisawa et al., 2008; Castaño et al., 2007) or CK1 $\varepsilon$ (Casagolda et al., 2010). We recently found that p120-catenin also plays an essential role in the early responses to Wnt3a ligand because it provides the platform for the interaction of CK1 $\varepsilon$ with the Wnt3a receptor complex and is necessary for Dvl2 and LRP5/6 phosphorylation (Casagolda et al., 2010). At later times, Wnt3a promotes the CK1 $\varepsilon$-dependent, CK1 $\alpha$-catalyzed phosphorylation of p120-catenin at Ser268 and 269, decreasing p120-catenin interaction with E-cadherin and thus releasing the catenin from the receptor complex (Casagolda et al., 2010; Del Valle-Pérez et al., 2011a). This E-cadherin-unbound p120-catenin plays additional roles in Wnt signaling because it interacts with Kaiso, preventing the inhibition by this factor of $\beta$-catenin/Tcf-4 complex (Del Valle-Pérez et al., 2011b). In this article, we show that p120-catenin, when released from E-cadherin, exerts an additional action in the Wnt response. Using SW-480 cells, a cell line deficient in $\beta$-catenin degradation that unveils Wnt effects on $\beta$-catenin other than protein stabilization, and HEK-293 cells, widely used to analyze the Wnt pathway, we demonstrate that p120-catenin participates in the stimulation of Rac1 activity, binding directly to this protein. In addition we show that Vav2 also binds to p120-catenin and is required for Rac1 activation and for $\beta$-catenin translocation to the nucleus.

\section{Results}

Go to:

\section{p120-catenin is required for transient Rac1 activation by Wnt3a}

We have analyzed Rac1 stimulation by Wnt3a using two cellular systems: HEK-293 fibroblasts, widely used to study this signaling pathway, and SW-480 colon cells that, although mutant in APC and therefore deficient in $\beta$-catenin degradation, are totally competent for other signals triggered by this stimulus (Casagolda et al., 2010; Del Valle-Pérez et al., 2011a; Del Valle-Pérez et al., 2011b). Upon Wnt3a addition, Rac1 became stimulated in both cell lines (Fig. 1A,B). Upregulation in Rac1 activity was transient; it was maximal at 2 hours after the addition of the stimulus and returned to the basal levels by 15 hours (Fig. 1A,B). No significant differences were detected between the two cell lines; in both cases Rac1 activity was $\sim 3$-fold higher at 2 hours of Wnt addition. Like other earlier responses, p120-catenin was required for Rac1 stimulation by Wnt3a; as shown in Fig. 1C,D, Rac1 activity was not sensitive to Wnt3a treatment in p120-catenin-depleted cells, contrarily to what happened in control cells. Basal Rac1 activity was also lower in p120-depleted cells than in control cells (ig. 1C,D).

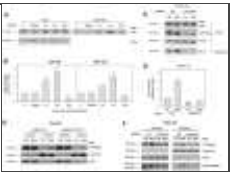

Fig. 1.

Wnt-induced Rac1 activation and nuclear $\beta$-catenin translocation are dependent on p120-catenin. (A) SW-480 cells treated with control or Wnt3a-conditioned medium for the indicated time were lysed and active Rac1 was precipitated using a GST-PAK ...

Other Wnt3a responses downstream of Rac1 activation were also affected by p120-catenin depletion. Wnt-induced JNK2 phosphorylation, catalyzed by Rac1-dependent PAK kinase (Wu et al., 2008), was not observed in p120-catenin deficient cells (supplementary material Fig. S1). The increase in nuclear $\beta$-catenin promoted by Wnt3a was also prevented in p120-catenin-depleted cells. These effects were observed in SW-480 ( Fig. 1E) and HEK-293 cells (Fig. 1F). As shown in Fig. 1E, and also supported by previous determinations of $\beta$-catenin transcriptional activity (Casagolda et al., 2010), SW-480 cells responded to Wnt3a with an increase in nuclear $\beta$-catenin despite the fact that they are deficient in $\beta$-catenin degradation, demonstrating that Wnt effects are not limited to $\beta$-catenin stabilization. Also, in accordance with previous observations indicating a role for Rac1 in $\beta$-catenin translocation to the nucleus (Wu et al., 2008), transfection of an inactive Rac1 mutant (RacN17) that works as a dominant negative lowered the nuclear $\beta$-catenin levels both in control and especially in Wnt3a- 
treated cells (supplementary material Fig. S2A). On the contrary, transfection of an active Rac1 mutant (RacV12) upregulated the amount of nuclear $\beta$-catenin in SW-480 cells (supplementary material Fig. S2B). This effect was not sensitive to p120-catenin downregulation (supplementary material Fig. S2B), indicating that Rac1 acts on $\beta$-catenin nuclear translocation downstream of p120-catenin.

\section{Vav2 interacts with p120-catenin and is required for Rac1 activation by Wnt3a}

Vav2 is a Rac1 activator widely expressed in epithelial cells (Bustelo, 2001). We detected Vav2 association to Rac1 by co-immunoprecipitation (coIP); the presence of Rac1 in Vav2-immunocomplexes was upregulated after 2 hours of incubation with Wnt3a, concomitantly with Rac1 activation and approximately to the same extent ( Fig. 2A, see also below). We also detected a simultaneous increase in the association of Vav2 with p120-catenin, an interaction that has been previously described by other authors (Noren et al., 2000). p120-catenin binding to Rac1 and Vav2 was also verified by an inverse coIP: both Rac1 and Vav2 were detected in p120-cateninimmunocomplexes (Fig. 2B). As above, the association was increased two hours after Wnt3a incubation, a time when p120-catenin has been released from its interaction with E-cadherin (Fig. 2B) (see also Casagolda et al., 2010; Del Valle-Pérez et al., 2011a). Disruption of E-cadherin-p120-catenin interaction by Wnt was also detected by the inverse co-immunoprecipitation (supplementary material Fig. S3).

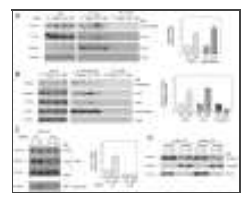

Fig. 2.

Wnt-induced Rac1 activation is dependent on Vav2 interaction with p120-catenin. Vav2 (A) and p120-catenin (B) were immunoprecipitated from $500 \mu \mathrm{g}$ SW-480 whole-cell extracts treated with control or Wnt3aconditioned medium for the times ...

RNA interference experiments demonstrate the requirement of Vav2 for Rac1 activation. As shown in Fig. 2C, activation of Rac1 by Wnt3a was totally prevented in Vav2-depleted cells. Rac1-downstream events, such as Wnt3a-stimulated $\beta$-catenin translocation to the nucleus (Fig. 2D) or JNK2 phosphorylation (supplementary material Fig. S4) in SW-480 cells were also inhibited by Vav2 depletion.

\section{Rac1 and Vav2 interact with p120-catenin and are regulated by tyrosine and serine phosphorylation}

Surprisingly, p120-catenin downregulation affected basal Rac1-Vav2 interaction (Fig. 3A), suggesting that this catenin facilitates the association of these two proteins. Alike another member of the Rac1 family, RhoA (Yanagisawa et al., 2008; Castaño et al., 2007), Rac1 interacts with p120-catenin (Orlichenko et al., 2010). Rac1-p120-catenin interaction was detected by pull-down assays performed using recombinant proteins, either with GST-p120-catenin (Fig. 3C; supplementary material Fig. S5) or GST-Rac1 (Fig. 3D) as baits. Association was not significantly different for nucleotide-unloaded, GTP- or GDP-bound Rac1 (supplementary material Fig. $\underline{\mathrm{S}} \mathrm{B})$. This differs from the p120-catenin-RhoA interaction that predominantly involves the GDP-bound inactive form of RhoA (Castaño et al., 2007). Mapping of the p120-catenin binding sequence using different protein fragments identified two different elements, placed within amino acids 1-234 and 350-911, capable of associating with Rac1 (supplementary material Fig. S5C,D). Therefore, p120-catenin isoform 3, corresponding to amino acids 102-911, presented lower binding to Rac1 than the full-length p120-catenin, isoform 1

(supplementary material Fig. S5C,D). One of the elements involved in Rac1 binding is placed in the p120-catenin armadillo repeats (comprising amino acids 347-820), the domain required for E-cadherin interaction. However, Rac1 did not interfere with p120-catenin association to E-cadherin in in vitro experiments: neither an E-cadherin fragment corresponding to the cytosolic domain (cytoE-cadh) competed with Rac1 binding to GST-p120-catenin nor recombinant Rac1 inhibited the association of cytoE-cadh to the same fusion protein (supplementary material Fig. S5E,F). Another member of the p120-catenin family, ARVCF, also bound Rac1 (supplementary material Fig.

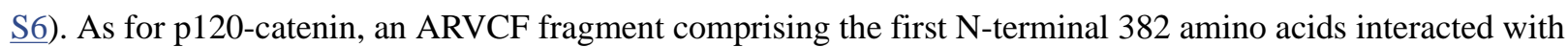
Rac1, although the binding was lower than with the full-length protein.

Fig. 3.

The direct interaction of p120-catenin with Rac1 and Vav2 is regulated by p120-catenin phosphorylation. (A) Vav2 was immunoprecipitated from $500 \mu \mathrm{g}$ of control and p120-catenin (p120-cat)-depleted SW-480 cell extracts. Protein complexes ... 
Vav2-pi20-catenin association was also studied using pull-down assays. Distinct from Rac1, binding of Vav2 to p120-catenin occurs in the first 390 amino acids without an additional interaction within the armadillo domain (supplementary material Fig. S7A,B). Given that amino acids 1-234 did not bind Vav2, the binding element putatively resides within amino acids $234-390$ of p120-catenin. As for Rac 1, p120-catenin isoform 1 binds to Vav2 better than p120-catenin isoform 3 (102-911) (supplementary material Fig. S7A,B). ARVCF also associates with Vav 2 through its first 382 amino acids (supplementary material Fig. S6). Recombinant Vav2 protein was also pulled down by GST-p120-catenin (supplementary material Fig. S7C, see also below), demonstrating that the interaction is direct. Binding of Rac1 to p120-catenin did not prevent the interaction of this protein with Vav2 (supplementary material Fig. S7C).

The N-terminal domain of p120-catenin (amino acids 1-347) is phosphorylated by several protein kinases. Among these, Src and Fyn modify tyrosines 112, 217 and others (Mariner et al., 2001; Piedra et al., 2003; Castaño et al., 2007), whereas the serine kinase CK1 phosphorylates Ser268, 269 and others (Casagolda et al., 2010). Modification of these two Ser residues significantly inhibits E-cadherin binding (Casagolda et al., 2010; see also below). We thus tested if p120-catenin phosphorylation by these kinases affected Vav2 or Rac1 binding. As shown in Fig. 3B, CK1 phosphorylation increased the binding of both proteins to p120-catenin; conversely, phosphorylation by Src and Fyn downregulated it. This decrease was also evident when p120-catenin was simultaneously phosphorylated by CK1 and Src/Fyn. As a control, E-cadherin-p120-catenin association was regulated as expected: diminished by CK1 and increased by Src and Fyn (Roura et al., 1999; Casagolda et al., 2010).

The relevance of the different modified amino acids in p120-catenin interaction with Rac1 and Vav2 was investigated. A p120-catenin mutant mimicking phosphorylated Ser268 and Ser269 (S268,269D) behaved similar than the wild-type protein with respect to the association with Rac1 (Fig. 3C,D,F) and Vav2 (Fig. 3E,F), indicating that CK1 effects on Vav2 and Rac1 are mediated by the modification of other p120-catenin residues. On the contrary, two Tyr-Glu mutants in p120-catenin, Y112E and Y217E, presented a downregulated interaction with Rac1 and Vav2. Y112E mutation slightly decreased p120-catenin association with Rac1 (Fig. 3D,F) whereas remarkably inhibited its binding to Vav2 (Fig. 3E,F); the Y217E mutant showed the same interaction with Vav2 as the wild-type protein, although binding to Rac1 was severely inhibited (Fig. 3D-F). The corresponding Tyr-Phe mutants (Y112F or Y217F) presented an unaltered binding to Vav2 and Rac1 than the wild-type p120-catenin (not shown). Therefore, these results indicate that CK1 and Src/Fyn phosphorylation contrarily regulates Rac1 and Vav2 binding to p120-catenin, and that the interaction of these two proteins is differently affected by modification of specific tyrosine residues in p120-catenin.

We verified if this phosphorylation-dependent regulation in the interaction of p120-catenin with Rac1 and Vav2 was also observed in vivo. As shown earlier and also in Fig. 3G, Wnt3a enhances p120-catenin interaction with Rac1 and Vav2 in SW-480 cells. This increase was accompanied by p120-catenin phosphorylation by CK1 $\alpha$, determined analyzing Ser268 modification (Del Valle-Pérez et al., 2011a; Del Valle-Pérez et al., 2011b). Conversely, tyrosine phosphorylation on p120-catenin was decreased by Wnt3a (Fig. 3G). This Wnt-dependent downregulation in PTyr was accompanied by a lower association of p120-catenin to Fyn, because the amount of this tyrosine kinase co-immunoprecipitating with p120-catenin was decreased upon 2 hours of Wnt3a addition ( Fig. 3H). Similar results were obtained in HEK293 cells (not shown). The downregulation in the tyrosine kinase binding to p120-catenin is likely to be the consequence of phosphorylation by CK1 because in vitro phosphorylation of recombinant p120-catenin by this protein kinase decreased its interaction with Fyn (Fig. 3I). In accordance with these results, the Tyr phosphorylation of specific residues in p120-catenin was detected mainly in the protein associated to the membrane and was downregulated upon the Wnt-induced release of p120-catenin to the cytosol (supplementary material Fig. S8).

\section{p120-catenin activates Rac1 activity}

Next we transfected the different p120-catenin forms used in this study into SW-480 and HEK-293 cells and determined their effects on Rac1 activity. In accordance to previous reports (Noren et al., 2000; Grosheva et al., 2001), both the full-length p120-catenin and a shorter construct, corresponding to isoform 3, increased Rac1 activity, as detected by pull-down assays with GST-PAK (Fig. 4A,B). Although they were similarly expressed, p120-catenin 1 (amino acids 1-911, indicated in the figures as WT) was slightly more active than p120-catenin 3 (102-911), particularly in HEK-293 cells. Other deletion mutants such as the 1-234 or 350-911 fragments, 
capable of binding Rac1 with low affinity but not Vav2, did not stimulate Rac1 activity (Fig. 4A,B). The Y112E p120-catenin mutant, partially defective in Rac1 association and totally unable to interact with Vav2, and the Y217E mutant, capable of associating with Vav2 but not with Rac1, were inactive in these assays. Y112F or Y217F mutants activated Rac1 as the wild-type p120-catenin (not shown). In accordance with the involvement of Rac1 in $\beta$-catenin nuclear import, whereas wild-type p120-catenin enhanced the $\beta$-catenin accumulation in the nucleus in Wnt3a-treated cells, the Y112E and Y217E mutants were unable to do so. Similar results were obtained in HEK-293 (Fig. 4C,D) or SW-480 cells (Fig. 4E,F). These results suggest that the interaction of p120-catenin with Vav2 and Rac1 is required for p120-catenin to stimulate Rac1 activity.

Fig. 4.

Only p120-catenin mutants able to interact with Rac1 and Vav2 induce

Rac1 activation. (A) GST-PAK pull-down assays were performed in

SW-480 cell extracts overexpressing either GFP-p120-catenin (p120-cat)

wild-type (wt) isoforms 1 or $3, \ldots$

We also analyzed the capability of the different p120-catenin mutants used in our study to overcome the effect of p120-catenin depletion on Rac1 activation by Wnt3a. We carried these assays ectopically expressing different GFP-p120-catenin proteins not targeted by the shRNA used to deplete endogenous p120-catenin. First we analyzed, whether the different p120-catenins rescued early responses to Wnt3a. As previously shown (Casagolda et al., 2010; ,Del Valle-Pérez et al., 2011a; see also Introduction), p120-catenin is essential for CK1 $\varepsilon$ activation, one of the initial responses to Wnt3a; therefore it is also required for later signals such as p120-catenin phosphorylation in Ser268 and 269 and $\beta$-catenin stabilization. As shown in Fig. 5A, the interaction of both the endogenous p120-catenin and the ectopically expressed GFP-p120-catenin with E-cadherin was disrupted by Wnt3a. The Y112E and Y217E mutants behave as the wild-type protein indicating that the Tyr mutants do not affect the early responses to Wnt signaling. As previously reported (Casagolda et al., 2010), the phosphorylationdeficient S268,269A p120-catenin remained associated to E-cadherin after Wnt stimulation.

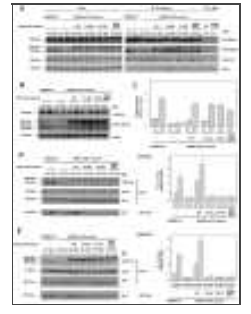

Fig. 5.

p120-catenin mutants unable to interact with Rac1 and Vav2 compromise Rac1 activation but not earlier steps of Wnt signaling. (A)

E-cadherin was immunoprecipitated from control and p120-catenin (p120cat)-depleted SW-480 cells overexpressing GFP-p120-catenin ...

This conclusion was supported by the determination of two other parameters. Wnt3a also induced a downregulation in $\beta$-catenin-E-cadherin interaction (Dupre-Crochet et al., 2007; Casagolda et al., 2010). p120-catenin depletion prevents the disruption of this association (Fig. 5A). Transfection of GFP-p120-catenin, either wild type, Y217E, Y112E or S268,269A restored the sensitivity of $\beta$-catenin-E-cadherin binding to Wnt3a (Fig. 5A).

Finally, Wnt3a increased the total levels of $\beta$-catenin, a stimulation that was also prevented by 120 -catenin depletion. This assay was carried out in HEK-293 cells that differently to SW-480 present an intact $\beta$-catenin degradation complex and respond to Wnt3a increasing $\beta$-catenin levels. Transfection of wild-type GFP-p120catenin or the Y217E, Y112E and, to a lower extent, S268,269A mutants enabled Wnt3a to accumulate $\beta$-catenin (Fig. 5B,C).

Once we determined that Y217E, Y112E or S268,269A were able to restore initial Wnt responses in p120-catenin-depleted cells, we analyzed their role in promoting Rac1 activation. As shown in Fig. 5D,E, contrarily to the wild-type protein, none of the three p120-catenin mutants was capable to recover Rac1 activation by Wnt3a, neither in SW-480 nor in HEK-293 cells. These results further indicate that the interaction of p120-catenin with Rac1 and Vav2, inhibited in Y217E and Y112E mutants, is necessary for Rac1 activation. They also demonstrate that p120-catenin release from E-cadherin is also required because the S268,269A mutant did not rescue the effect of p120-catenin depletion on Rac1 activation. 
We further investigate the action of E-cadherin on Rac1 activation. In these experiments we used p120-catenin S268,269D and S268,S269A mutants that are deficient for E-cadherin binding or show a Wnt-independent interaction, respectively. When overexpressed, both mutants activated Rac1 (Fig. 6A,B, compare lane 1 and 4 or 5). Co-transfection of E-cadherin repressed the stimulation caused by p120-catenin S268,269A but did not affect that promoted by S268,269D (Fig. 6A,B, lanes 2 and 3). Similar results were obtained in HEK-293 (Fig. 6A,B) and SW-480 cells (Fig. 6B). Like the wild-type protein (Castaño et al., 2007), when ectopically expressed in HEK-293 cells both p120-catenin mutants were detected in the cytosolic and membrane fractions whereas Vav2 was only detected in the cytosol (Fig. 6C). E-cadherin coexpression translocated S268,269A mutant to the membrane fraction but did not modify the distribution of the S268,269D protein, as expected for a mutant unable to interact with E-cadherin (Casagolda et al., 2010).

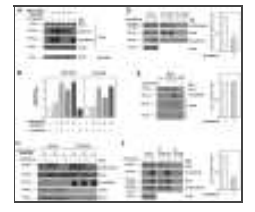

Fig. 6 .

Only cytosolic p120-catenin interacts with Vav2 and activates Rac1. (A) HEK-293 cells were transfected with GFP-p120-catenin (p120-cat) isoform 1 point mutants S268,269A, S268,269D and E-cadherin or the empty vector phrGFP. GST-PAK ...

Therefore, E-cadherin retains p120-catenin in the membrane putatively preventing its interaction with Vav2. Co-immunoprecipitation experiments indicated that E-cadherin overexpression decreased p120-catenin levels in Vav2-immunocomplexes (Fig. 6D), demonstrating that E-cadherin downregulates Vav2-p120-catenin interaction. However, E-cadherin by itself does not inhibit Vav2 binding to p120-catenin, because addition of a recombinant fragment corresponding to the cytosolic domain of E-cadherin (cytoE-cadh) did not downregulate the amount of Vav2 pulled down by GST-p120-catenin, in assays performed with recombinant proteins. Thus, p120-catenin can simultaneously bind to both proteins (Fig. 6E). The negative effect of E-cadherin on Vav2-p120-catenin interaction was also verified immunoprecipitating p120-catenin. Ectopic E-cadherin expression decreased Vav2 protein present in the p120-catenin-immunocomplex (Fig. 6F). Concomitantly, E-cadherin upregulated tyrosine phosphorylation on p120-catenin (Fig. 6F), providing an explanation for the inhibition of Vav2-p120-catenin binding because, as presented above (see Fig. 3B), tyrosine phosphorylation of p120-catenin hampers this interaction.

\section{p120-catenin mutants deficient in Vav2 or Rac1 binding do not rescue gastrulation defects in Xenopus embryos}

Depletion of p120-catenin in Xenopus laevis affects normal embryo development (Fang et al., 2004). We used an established morpholino (MO) to deplete X. laevis p120-catenin. As shown in supplementary material Fig. S9A, this MO efficiently decreased p120-catenin protein levels when injected into both blastomeres at the two-cell stage. At the morpholino dose employed, between 40 and $70 \%$ of the injected embryos displayed aberrant gastrulation in different assays (Fig. 7). This phenotype was rescued upon ectopic expression of myc-tagged murine p120-catenin (a transcript that lacks the morpholino binding sequence) (Fig. 7A,B). Injection of constitutive-active Rac1 (CA-Rac1) also significantly decreased the aberrant gastrulation phenotype (Fig. 7E,F) indicating that p120-catenin depletion was affecting Rac1 activity. p120-catenin mutant Y112F was as effective as the wild-type form in rescuing the gastrulation defects (Fig. 7A,B); on the contrary, Y112E or Y217E failed to rescue in these assays (Fig. 7B,F), although they were expressed at similar levels than the other p120-catenin forms (supplementary material Fig. S9B,C). The p120-catenin mutant S268,269A, unable to be released from E-cadherin, was also inactive in these assays, whereas p120-catenin Ser268,269D significantly rescued p120-catenin deficiency (Fig. 7C,D), despite all these mutants being similarly expressed (supplementary material Fig. S9C). As observed for the p120-catenin depleted embryos, CA-Rac1 injection appreciably recovered the wild-type phenotype in the Y112E or Y217E rescues (Fig. 7E,F). The results were significant for Y217E mutants and not for $\mathrm{Y} 112 \mathrm{E}(P=0.075)$ probably due to the high $\mathrm{SD}$, although a partial recovery of the gastrulation delay was observed in all the experiments performed.

Fig. 7.

p120-catenin point mutants unable to bind Rac1 or Vav2 do not rescue gastrulation defects caused by p120-catenin depletion in Xenopus embryos. Embryos at the two-cell 


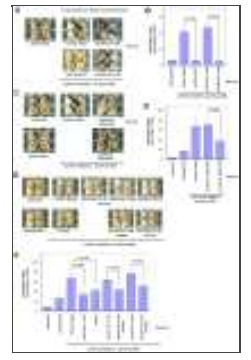

stage were injected with a control morpholino or a combination of xp120 mi

\section{Discussion}

Go to:

Recent results suggest that E-cadherin-dependent adherens junctional complexes are closely associated with Wnt receptors. Besides $\beta$-catenin, another E-cadherin associated protein, p120-catenin is also required for canonical Wnt signaling. Through its interaction with E-cadherin (or N-cadherin in mesenchymal cells), p120-catenin associates with the LRP5/6 coreceptor and enables the binding to this complex of CK1 1 , a protein kinase constitutively associated with p120-catenin. This catenin is required for CK1 $\varepsilon$ activation and for the initial responses to Wnt3a, such as Dvl2 or LRP5/6 phosphorylation (Casagolda et al., 2010; Del Valle-Pérez et al., 2011a; Del Valle-Pérez et al., 2011b). A current model depicting our vision of this process is presented in Fig. 8. Panel A shows the protein complexes in nonstimulated cells, whereas panel B represents the formation of the Wnt receptor complex upon binding of the ligand.

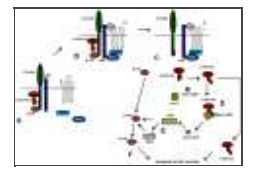

Fig. 8.

\section{Model for Wnt-induced Rac1 activation through p120-catenin and}

Vav2. In nonstimulated cells (A), p120-catenin (p120-cat) is bound to E-cadherin (E-cadh), and through this protein to LRP5/6. Upon binding of the Wnt factor $(\mathbf{B})$, a receptor complex is formed ...

At longer times, p120-catenin is released from the E-cadherin-Wnt receptor complex upon its phosphorylation at Ser268 and 269 by CK1 $\alpha$ (Fig. 8C) (Casagolda et al., 2010). Separation from E-cadherin enables p120-catenin to translocate to the cytosol because its presence in the membrane is dependent on its interaction with cadherins. Moreover, p120-catenin phosphorylation by CK1 decreases the interaction with tyrosine kinases active on this catenin, such as Fyn, which binds to the same region of the $\mathrm{N}$ terminus, and remains associated to the membrane. As a consequence, upon Wnt stimulation, p120-catenin undergoes tyrosine dephosphorylation. This allows p120-catenin binding to Rac1 and Vav2, because p120-catenin tyrosine phosphorylation hinders the interaction with these proteins. Our results help to explain why E-cadherin expression inhibits Rac1 activity (Soto et al., 2008) and prevents Vav2 and Rac1 binding to p120-catenin. Although E-cadherin binding does not directly inhibit the association of these two factors with p120-catenin, its action recruiting this catenin to the membrane promotes its tyrosine phosphorylation by membrane-associated tyrosine kinases and, therefore indirectly disrupts p120-catenin binding to Vav2 or Rac1, markedly constrained by this modification. The results shown in Fig. 6F, demonstrating that E-cadherin enhances p120-catenin PTyr, further support this model. Moreover, the influence of E-cadherin (and probably other cadherins) in Rac1 control explains the different basal activity of this GTPase in different cell lines.

Ser-phosphorylated, Tyr-dephosphorylated p120-catenin binds to Vav2 and Rac1 (Fig. 8D). These two proteins associate preferentially to the $\mathrm{N}$-terminal domain, although the armadillo repeats are also relevant for the full Rac1 binding. Association with p120-catenin enhances Vav2 activity towards Rac1. There are several possibilities to explain this fact. First, it is possible that it can potentiate the tyrosine phosphorylation of Vav2 by a tyrosine kinase, still associated to p120-catenin. However, this hypothesis is unlikely because we did not detect an increase in Vav2 tyrosine phosphorylation upon Wnt stimulation. Alternatively and more likely, it is possible that the interaction frees the Vav2 catalytic Dbl homology domain from the coordinated auto-inhibition by the Acidic and Calponin homology domains (Yu et al., 2010). Thus, p120-catenin interaction would mimic the effects of Vav2 phosphorylation in the Acidic domain. It is noteworthy that a previous report has demonstrated that Vav1 can be stimulated by physical interaction with p67phox protein in phagocytic leukocytes (Ming et al., 2007). Just as p120-catenin, p67phox interacts with both Vav1 and Rac1, enhances Vav1/Rac1 association and stimulates Vav1 activity without increasing tyrosine phosphorylation. 
Upon activation, GTP-bound Rac1 stimulates PAK protein kinase that in turn phosphorylates and activates JNK2 (Wu et al., 2008) (Fig. 8E). This protein kinase modifies two residues in $\beta$-catenin, Ser191 and 605, necessary for the translocation of this protein to the nucleus (Fig. 8F). Alternatively, or additionally, PAK1 also phosphorylates directly $\beta$-catenin at Ser675, contributing to its stabilization (Zhu et al., 2012). This $\beta$-catenin might come from the pool associated to E-cadherin and released upon phosphorylation of this protein by CK1 $\alpha$ (Dupre-Crochet et al., 2007), or upon $\beta$-catenin modification by tyrosine kinases (Piedra et al., 2003). It might also come from other signaling pools of $\beta$-catenin, normally degraded through the action of the $\beta$-catenin destruction complex either in the cytosol or in the membrane (Maher et al., 2009). In any case, Wnt blocks $\beta$-catenin degradation by these complexes and stimulates further $\beta$-catenin phosphorylation increasing Rac1-dependent PAK and JNK2 activities. Besides this role in Rac1 activation, E-cadherin-unbound p120-catenin plays an additional function in Wnt signaling because it translocates to the nucleus and releases the inhibition caused by Kaiso on the expression of several target genes (Del Valle-Pérez et al., 2011b; Kelly et al., 2004; Kim et al., 2004; Park et al., 2006).

The experimental data are compatible with this model. However, some limitations of results based on cell lines must be taken into account. For instance, it remains to be established whether other proteins similar to p120-catenin promote the same function on Rac1 stimulation. Our data indicate that ARVCF binds to both Rac1 and Vav2 suggesting that it might also be involved in Rac1 activation. It is likely that the contribution of p120-catenin and ARVCF (and perhaps other members of the family) depends on their relative protein levels in different cell lines. Another issue for further research consists in the action of E-cadherin and N-cadherin on p120-catenin function. Although preliminary observations indicate that both cadherins bind p120-catenin with a similar affinity, it is possible that the presence of tyrosine kinases in their complexes might vary, affecting differently the function of p120-catenin on Rac1.

Finally, our model proposes that p120-catenin is essential for the activation of $\beta$-catenin transcriptional activity by Wnt but is not sufficient to stimulate this activity, because it does not upregulate $\beta$-catenin stability. For instance, in HEK-293 cells, p120-catenin does not significantly increase nuclear $\beta$-catenin (not shown). Accordingly, in Xenopus embryos ventral overexpression of p120-catenin does not induce the formation of duplicate axial structures, which differs from what happens with $\beta$-catenin (Paulson et al., 1999). It is likely that detection of these structures would require the co-injection of p120-catenin and sub-phenotypic doses of $\beta$-catenin.

In any case, our results reinforce the idea that proteins associated with adherens junctions, in addition to, but distinct from $\beta$-catenin, contribute to Wnt signaling; particularly, p120-catenin plays a multiple role in the activation of this signaling pathway. According to our data, one of these p120-catenin functions, activation of Rac1, is finely controlled through serine and tyrosine phosphorylation. It remains to be established whether a similar mechanism also participates in the p120-catenin-mediated activation of Rac1 by other stimuli (Yanagisawa et al., 2008; Johnson et al., 2010).

Materials and Methods Go to:

Cell culture

Cell lines were obtained from our Institute Cell Bank. Control L fibroblasts or cells stably transfected with a plasmid encoding Wnt3a were obtained from the ATCC and cultured in medium containing $0.4 \mathrm{mg} / \mathrm{ml} \mathrm{G}-418$. When indicated, cells were treated with conditional medium from control or Wnt3a expressing cells for the indicated time.

\section{Antibodies}

The following antibodies were used in this study. Monoclonal antibodies (mAbs): $\beta$-catenin (BD Biosciences, ref. 65394), p120-catenin (Armadillo domain, BD Biosciences, ref. 610134), p120-catenin (N-tail, BD Biosciences 610921), phosphoSer268 in p120-catenin (BD Biosciences 558383), E-cadherin (BD Biosciences, ref. 610182), Rac1 (BD Biosciences, ref. 610650), Pyr K (Chemicon AB-1235), Lamin B (Abcam Ab 16048), Vav2 (Santa Cruz sc-20803), phosphotyrosine (PTyr) (BD Biosciences, ref. 610000), Fyn (BD Biosciences, ref. 610164), JNK2 (Santa Cruz N-18: sc-827), phosphoJNK2 (PJNK2) (Millipore 07-175), and Myc (clone 9E10 from DSHB). Polyclonal antibodies: GST (GE Healthcare, ref. 27-4577-01); GAPDH (Santa Cruz clone FL-335: sc-25778) and Xenopus p120-catenin (C-tail, Fang et al., 2004). 


\section{Preparation of DNA constructs}

The bacterial expression plasmid pGEX6P, encoding a glutathione $S$-transferase (GST) fused to Rac1 was obtained from pGEX-4T3-Rac1 (provided by Dr Servitja, CEK-IDIBAPS, Barcelona, Spain) by standard cloning procedures using the flanking EcoRI and BamHI sites present in the polylinker of pGEX-6P3 plasmid. The pGEX-6P2-Vav2 was generated from pECFL-HA-Vav2 provided by Dr X. Bustelo (CIC, Salamanca, Spain) by digestion with EcoRI and cloned into the plasmid digested with the same enzyme. The generation of pGEX-6P3p120-catenin wild type and the deletion and point mutants have been previously described (Castaño et al., 2007; Casagolda et al., 2010). The preparation of the pGEX-6P1-p120-catenin (1-350) and pGEX-6P3-p120-catenin (1-390) constructs were generated from pGEX-p120-catenin (1-911) by using oligonucleotides corresponding to the nucleotide sequences 1 to 18 in both cases and 1041 to 1055 or 1156 to 1170 that contained EcoRI and EcoRV or XhoI sites, respectively. Amplification products were cloned into pGEX-6P1 or pGEX-6P3 digested with the EcoRI and SmaI or XhoI enzymes, respectively. To generate pGEX-6P-ARVCF wild type, total RNA extracts from HCT116 cells were obtained and cDNA was generated using RT-Roche Single Strand kit. A DNA fragment that corresponds to human ARVCF was amplified by PCR using the following sense and antisense primers, respectively: 5'-CCAGAATTCATGGAGGACTGCAA-3' and 5'-CGAGTCGACCTAGACCCAGGAATC-3' that contained EcoRI and SalI sites at their 5'-ends, respectively. The 3-kilobase amplification fragment was digested with EcoRI and SalI and cloned in the same sites of pGEX-6P1 plasmid. The absence of mutations in this fragment was verified by sequencing. The pGEX-6P-ARVCF (1-382) was prepared by PCR amplification from the pGEX-6P-ARVCF and digesting the insert and vector with BamHI/EcoRI. The1.2-kilobase fragment obtained was re-ligated in to the vector.

The use of eukaryotic expression plasmid (phrGFP-N1) codifying for GFP-p120-catenin (102-911), GFP-p120catenin (1-911), and GFP-p120-catenin (1-911) point mutants Y112E, Y112F, Y217E, Y217F, S268-269A, S268-269D, has been reported (Castaño et al., 2007; Casagolda et al., 2010). pEGFP-C1-RacV12 was provided by Dr F. Sánchez Madrid (Hospital de la Princesa, Madrid, Spain). The deletion mutant phrGFP-p120-catenin (8-390) was generated from phrGFP-p120-catenin (1-911). Oligonucleotides corresponding to nucleotide sequences 1152 to 1170 and 2716 to 2736 that contained EcoRV site were used to amplify the 8-390 region of p120-catenin. The 5.5-kbp amplification product was digested with EcoRV and ligated. EcoRV site was eliminated. Absence of mutations in all generated constructs was checked by sequencing.

The pCS2 constructs used for in vitro transcription and later injection in X. laevis embryos were generated from phrGFP-p120-catenin constructs described above and were digested with EcoRI and SacII and cloned into the plasmid digested with the same enzymes. Verification of constructs expression was assessed by transfection in mammalian cells. The constitutively active Rac1 (CA-RAc1) construct has been previously reported (Fang et al., 2004).

\section{Expression of recombinant proteins and pull-down assays}

GST-fusion proteins were expressed in E. coli and purified by affinity chromatography with GlutathioneSepharose as described (Solanas et al., 2004). When required, GST was removed by cleaving with Pre-Scission protease (Amersham Biosciences). Phosphorylation of p120-catenin by recombinant CK1 (Sigma) or recombinant Src and Fyn (Upstate) was performed as reported (Castaño et al., 2007; Casagolda et al., 2010). Pull-down assays were performed using purified recombinant proteins fused to GST as described (Casagolda et al., 2010). Glutathione-Sepharose-bound proteins were analyzed by western blotting using specific antibodies. Immunoblots were analyzed using the SNAP protein detection system (Millipore). All binding assays were repeated three times.

\section{Cell transfection and selection of transfectants}

The generation of SW-480 or HEK-293 cells expressing with control or p120-catenin shRNA, has been described (Casagolda et al., 2010). Transient expression of ectopic proteins was achieved in 50\% confluent cells transfecting the indicated eukaryotic plasmid using either Lipofectamine 2000 (Invitrogen) or PEI (Polyethilamine, Polysciences, Inc.). Cells were analyzed after selection with $2 \mu \mathrm{g} / \mathrm{ml}$ puromycin. Human shRNA for Vav2 (TRCN0000048227) or p120-catenin (TRCN0000122988) were obtained from Sigma. SW-480 cells were infected with lentiviral particles containing a shRNA targeting Vav2 or p120-catenin. Infected cells were selected with puromycin at $2 \mu \mathrm{g} / \mathrm{ml}$. Control cells were infected with lentivirus bearing a nontargeting shRNA 
(clone SCH002; Sigma).

\section{Cellular subfractionation}

To separate nuclei form cytosol and membranes, cells were lysed in $50 \mathrm{mM}$ Tris $\mathrm{pH}$ 7.5, 1\% TX-100, $137 \mathrm{mM}$ $\mathrm{NaCl}$ and $10 \%$ Glycerol supplemented with protease inhibitors $(0.3 \mu \mathrm{M}$ Aprotinin, $1 \mu \mathrm{M}$ Leupeptin, $1 \mu \mathrm{M}$ Pepstatin, $1 \mathrm{mM}$ Pefabloc) and phosphatase inhibitors (10 mM NaF, $0.1 \mathrm{mM}$ sodium orthovanadate and $2.5 \mathrm{mM}$ $\beta$-glycerol-phosphate). Extracts were left on ice for 15 minutes and centrifuged for 15 minutes at $500 \mathrm{~g}$ and $4^{\circ} \mathrm{C}$. The supernatant contained the cytosol, membrane and cytoskeleton fraction. The pellet was lysed in half of the initial volume of the buffer adding SDS to a $0.5 \%$ final concentration. Extracts were sonicated for 5 seconds and centrifuged for 15 minutes at $16,000 \mathrm{~g}$ and $4^{\circ} \mathrm{C}$. The supernatant contained the nuclear fraction. Proteins were analyzed by western blot.

To separate cytosol from membranes, cells were lysed in $10 \mathrm{mM}$ Tris- $\mathrm{HCl} \mathrm{pH} 7.4,1 \mathrm{mM} \mathrm{MgCl}_{2}, 1 \mathrm{mM}$ EDTA supplemented with protease inhibitors ( $0.3 \mu \mathrm{M}$ Aprotinin, $1 \mu \mathrm{M}$ Leupeptin, $1 \mu \mathrm{M}$ Pepstatin, $1 \mathrm{mM}$ Pefabloc) and phosphatase inhibitors ( $10 \mathrm{mM} \mathrm{NaF}, 0.1 \mathrm{mM}$ sodium orthovanadate and $2.5 \mathrm{mM} \beta$-glycerol-phosphate). Cells were homogenized with a $25 \mathrm{G}$ needle 10 times using a $1 \mathrm{ml}$ syringe. $4 \mathrm{M} \mathrm{NaCl}$ was added to a final concentration of $0.15 \mathrm{M}$ and lysate was centrifuged for $10 \mathrm{~s}$ at $16,000 \mathrm{~g}$. The supernatant contained the cytosol and part of membranes. The pellet containing nuclei and remaining membranes was washed twice with PBS, resuspended with the same volume of $20 \mathrm{mM}$ Tris $\mathrm{pH} 7.5,100 \mathrm{mM} \mathrm{NaCl}, 1 \mathrm{mM}$ EDTA, $10 \mathrm{mM} \mathrm{MgCl} 2,1 \%$ Nonidet P-40 supplemented with protease and phosphatase inhibitors and centrifuged for 1 minute at $16,000 \mathrm{~g}$. The nuclear pellet was discarded and the supernatant contained the rest of solubilized membranes. The cytosolic supernatant was then ultracentrifuged for 40 minutes at $100,000 \mathrm{~g}$ at $4^{\circ} \mathrm{C}$ to separate membranes. The ultracentrifuged supernatant contained only the cytosolic fraction. Ultracentrifuged membranes were resuspended in the same volume of RIPA buffer and mixed with the supernatant with the solubilized membranes. For western blot analysis, the volume of cytosol was half of that for membranes.

\section{Immunoprecipitation assays}

Cell extracts were prepared by homogenizing cells in lysis buffer $(20 \mathrm{mM}$ Tris- $\mathrm{HCl} \mathrm{pH} \mathrm{7.6,100} \mathrm{mM} \mathrm{NaCl,} 10$ $\mathrm{mM} \mathrm{MgCl} 2,1 \mathrm{mM}$ EDTA and $0.5 \% \mathrm{NP}-40)$ supplemented with protease inhibitors $(0.3 \mu \mathrm{M}$ Aprotinin, $1 \mu \mathrm{M}$ Leupeptin, $1 \mu \mathrm{M}$ Pepstatin, $1 \mathrm{mM}$ Pefabloc) and phosphatase inhibitors (10 mM NaF, $0.1 \mathrm{mM}$ sodium orthovanadate and $2.5 \mathrm{mM} \beta$-glycerol-phosphate). After passing cells ten times through a $25 \mathrm{G}$ needle using a $1 \mathrm{ml}$ syringe, extracts were left on ice for 20 minutes and centrifuged at $14,000 \mathrm{~g}$ for 10 minutes at $4{ }^{\circ} \mathrm{C}$. Supernatants constituted the cell extracts. Proteins were immunoprecipitated from cell extracts (300 to $600 \mu \mathrm{g}$ ) using $2 \mu \mathrm{g} / \mathrm{ml}$ of the appropriate antibody or an irrelevant $\operatorname{IgG}$ as control for $16 \mathrm{~h}$ at $4{ }^{\circ} \mathrm{C}$. Precipitated material was removed by centrifugation at 12,000 $\mathrm{g}$ and the resulting supernatant was incubated for 120 minutes with $20 \mu \mathrm{l}$ of $\gamma$-bind G-Sepharose (GE Healthcare). Immunoprecipitates were washed three times with PBS 0.1\% NP-40 and bound proteins were eluted with electrophoresis sample buffer. Immunoprecipitated proteins were analyzed by western blot using specific mAbs.

\section{Rac1 activity assay}

Rac1 activity was determined in cells using specific pull-down assays for the activated form of this protein. Active Rac1 was affinity precipitated using the Rac1 binding domain of PAK. The fusion protein GST-PAK was purified from bacteria and bound to glutathione Sepharose beads to precipitate GTP-Rac1. Cells were lysed in RIPA buffer and centrifuged for 5 minutes at $16,000 \mathrm{~g}$. The supernatant was incubated at $4{ }^{\circ} \mathrm{C}$ with recombinant GST-PAK for 30 minutes. Samples were washed twice and bound proteins were analyzed by western blot.

\section{Xenopus laevis embryos and microinjection experiments}

$X$. laevis females were induced to lay eggs, which were fertilized and microinjected using published procedures (Fang et al., 2004). Morpholinos and RNA constructs were microinjected into the animal hemispheres of one- or two-cell cleavage-stage embryos. Injected embryos were cultured in $0.1 \times \mathrm{MMR}$ buffer $(100 \mathrm{mM} \mathrm{NaCl}, 2 \mathrm{mM}$ $\mathrm{KCl}, 2 \mathrm{mM} \mathrm{CaCl}_{2}, 5 \mathrm{mM}$ HEPES $5 \mathrm{mM} \mathrm{pH}$ 7.4) containing $50 \mu \mathrm{g} / \mathrm{ml}$ gentamycin until desired stages. The injection volume for morpholinos or RNAs was $20 \mathrm{nl}$ at the one-cell stage, $10 \mathrm{nl}$ per blastomere at the two-cell stage and beyond with the doses as follows: $40 \mathrm{ng}$ for p120-catenin morpholinos (20 ng each morpholino I and II) 
per blastomere (80 ng/embryo); $1 \mathrm{ng}$ per embryo for p120-catenin (1-911) wt, Y112E, Y112F, Y217E, S268,269D and S268,269DA for WB detection; 40 pg per embryo for p120-catenin wt, Y112E and Y112F rescues, 10 pg per embryo for p120-catenin Y217E rescues, 80 pg per embryo for p120-catenin S268,269D and S268,269A rescues and $1 \mathrm{pg}$ per embryo for CA-Rac rescues. The same rescue doses in each experiment were used for the control construct $\beta$-galactosidase. Gastrulation phenotypes, principally failure of blastopore closure, were visually scored at embryonic stages 11-12 using a standard binocular dissecting microscope (Zeiss Stemi DV4). Embryos were microinjected with capped mRNA synthesized in vitro (mMessage mMachine, Ambion). All pCS2-based constructs were linearized by using NotI before in vitro transcription. p120-catenin morpholinos were obtained from Gene Tools, LLC, and were reported previously (Fang et al., 2004; Kim et al., 2004).

\section{Ethics}

All animal experiments were performed according to the approved guidelines.

Supplementary Material Go to:

\section{Supplementary Material:}

Click here to view.

\section{Acknowledgments}

Go to:

We are grateful to Drs A.B. Reynolds, J.M. Servitja, F. Sánchez-Madrid and X. Bustelo for providing reagents. We also thank N. Ontiveros for excellent technical support and Dr D. Casagolda for helpful discussions.

Footnotes Go to:

\section{Funding}

This study was supported by grants awarded by the Ministerio de Ciencia e Innovación (MICINN) [grant numbers BFU2009-07578 to M.D., SAF2010-16089 to A.G.H.]; Asociación Española contra el Cáncer (AECC) and La Fundació La Marató de TV3 [reference 081731]. Partial support from the Instituto Carlos III [grant number RD06/0020/0040] and the Generalitat de Catalunya [grant number 2009SGR-121 and 2009SGR-585] is also appreciated. G.V. and M.V. were supported by predoctoral fellowships from the MICINN and M.C. by a postdoctoral fellowship from the Instituto Carlos III. P.D.M. was funded by the National Institutes of Health (NIH) [grant number RO1 M52112] and March of Dimes [grant number 1-FY-07-461-01]; and R.K.M. through NIH grants [grant numbers HD07325, DK082145]. Deposited in PMC for release after 12 months.

Supplementary material available online at http://jcs.biologists.org/lookup/suppl/doi:10.1242/jcs.101030/-/DC1

Bustelo X. R. (2001). Vav proteins, adaptors and cell signaling. Oncogene 20, 6372-6381 10.1038/sj.onc.1204780 [PubMed] [Cross Ref]

Casagolda D., Del Valle-Pérez B., Valls G., Lugilde E., Vinyoles M., Casado-Vela J., Solanas G., Batlle E., Reynolds A. B., Casal J. I. et al. (2010). A p120-catenin-CK1 $\varepsilon$ complex regulates Wnt signaling. J. Cell Sci. 123, 2621-2631 10.1242/jcs.067512 [PubMed] [Cross Ref]

Castaño J., Solanas G., Casagolda D., Raurell I., Villagrasa P., Bustelo X. R., García de Herreros A., Duñach M. (2007). Specific phosphorylation of p120-catenin regulatory domain differently modulates its binding to RhoA. Mol. Cell. Biol. 27, 1745-1757 10.1128/MCB.01974-06 [PMC free article] [PubMed] [Cross Ref]

Daniel J. M., Reynolds A. B. (1999). The catenin p120(ctn) interacts with Kaiso, a novel BTB/POZ domain zinc finger transcription factor. Mol. Cell. Biol. 19, 3614-3623 [PMC free article] [PubMed]

Del Valle-Pérez B., Arqués O., Vinyoles M., de Herreros A. G., Duñach M. 2011a). Coordinated action of CK1 isoforms in canonical Wnt signaling. Mol. Cell. Biol. 312877-2888 10.1128/MCB.01466-10 [PMC free article] [PubMed] [Cross Ref]

Del Valle-Pérez B., Casagolda D., Lugilde E., Valls G., Codina M., Dave N. et al. 2011b). Wnt controls the transcriptional activity of Kaiso through CK1 1 -dependent phosphorylation of p120-catenin. J. Cell Sci. 1242298-2309 10.1242/jcs.082693 [PubMed] [Cross Ref]

Dupre-Crochet S., Figueroa A., Hogan C., Ferber E. C., Bialucha C. U., Adams J., Richardson E. C., Fujita Y. 
(2007). Casein kinase 1 is a novel negative regulator of E-cadherin-based cell-cell contacts. Mol. Cell. Biol. 27, 3804-3816 10.1128/MCB.01590-06 [PMC free article] [PubMed] [Cross Ref]

Etienne-Manneville S., Hall A. (2002). Rho GTPases in cell biology. Nature 420, 629-635 10.1038/nature01148 [PubMed] [Cross Ref]

Fang X., Ji H., Kim S. W., Park J. I., Vaught T. G., Anastasiadis P. Z., Ciesiolka M., McCrea P. D. (2004). Vertebrate development requires ARVCF and p120 catenins and their interplay with RhoA and Rac. J. Cell Biol. 165, 87-98 10.1083/jcb.200307109 [PMC free article] [PubMed] [Cross Ref]

Grosheva I., Shtutman M., Elbaum M., Bershadsky A. D. (2001). p120 catenin affects cell motility via modulation of activity of Rho-family GTPases: a link between cell-cell contact formation and regulation of cell locomotion. J. Cell Sci. 114, 695-707 [PubMed]

Johnson E., Seachrist D. D., DeLeon-Rodriguez C. M., Lozada K. L., Miedler J., Abdul-Karim F. W., Keri R. A. (2010). HER2/ErbB2-induced breast cancer cell migration and invasion require p120 catenin activation of Rac1 and Cdc42. J. Biol. Chem. 285, 29491-29501 10.1074/jbc.M110.136770 [PMC free article] [PubMed] [Cross Ref]

Kelly K. F., Otchere A. A., Graham M., Daniel J. M. (2004). Nuclear import of the BTB/POZ transcriptional regulator Kaiso. J. Cell Sci. 117, 6143-6152 10.1242/jcs.01541 [PubMed] [Cross Ref]

Kim S. W., Park J. I., Spring C. M., Sater A. K., Ji H., Otchere A. A., Daniel J. M., McCrea P. D. (2004). Non-canonical Wnt signals are modulated by the Kaiso transcriptional repressor and p120-catenin. Nat. Cell Biol. 6, 1212-1220 10.1038/ncb1191 [PubMed] [Cross Ref]

Klaus A., Birchmeier W. (2008). Wnt signalling and its impact on development and cancer. Nat. Rev. Cancer 8, 387-398 10.1038/nrc2389 [PubMed] [Cross Ref]

Liu C., Li Y., Semenov M., Han C., Baeg G. H., Tan Y., Zhang Z., Lin X., He X. (2002). Control of beta-catenin phosphorylation/degradation by a dual-kinase mechanism. Cell 108, 837-847 10.1016/S0092-8674(02)00685-2 [PubMed] [Cross Ref]

MacDonald B. T., Tamai K., He X. (2009). Wnt/beta-catenin signaling: components, mechanisms, and diseases. Dev. Cell 17, 9-26 10.1016/j.devcel.2009.06.016 [PMC free article] [PubMed] [Cross Ref]

Maher M. T., Flozak A. S., Stocker A. M., Chenn A., Gottardi C. J. (2009). Activity of the beta-catenin phosphodestruction complex at cell-cell contacts is enhanced by cadherin-based adhesion. J. Cell Biol. 186, 219-228 10.1083/jcb.200811108 [PMC free article] [PubMed] [Cross Ref]

Mariner D. J., Anastasiadis P., Keilhack H., Böhmer F. D., Wang J., Reynolds A. B. (2001). Identification of Src phosphorylation sites in the catenin p120ctn. J. Biol. Chem. 276, 28006-28013 10.1074/jbc.M102443200 [PubMed] [Cross Ref]

Ming W., Li S., Billadeau D. D., Quilliam L. A., Dinauer M. C. (2007). The Rac effector p67phox regulates phagocyte NADPH oxidase by stimulating Vav1 guanine nucleotide exchange activity. Mol. Cell. Biol. 27, 312-323 10.1128/MCB.00985-06 [PMC free article] [PubMed] [Cross Ref]

Noren N. K., Liu B. P., Burridge K., Kreft B. (2000). p120 catenin regulates the actin cytoskeleton via Rho family GTPases. J. Cell Biol. 150, 567-580 10.1083/jcb.150.3.567 [PMC free article] [PubMed] [Cross Ref]

Orlichenko L., Geyer R., Yanagisawa M., Khauv D., Radisky E. S., Anastasiadis P. Z., Radisky D. C. (2010). The 19-amino acid insertion in the tumor-associated splice isoform Rac1b confers specific binding to p120 catenin. J. Biol. Chem. 285, 19153-19161 10.1074/jbc.M109.099382 [PMC free article] [PubMed] [Cross Ref]

Park J. I., Ji H., Jun S., Gu D., Hikasa H., Li L., Sokol S. Y., McCrea P. D. (2006). Frodo links Dishevelled to the p120-catenin/Kaiso pathway: distinct catenin subfamilies promote Wnt signals. Dev. Cell 11, 683-695 10.1016/j.devcel.2006.09.022 [PubMed] [Cross Ref]

Parri M., Chiarugi P. (2010). Rac and Rho GTPases in cancer cell motility control. Cell Commun. Signal. 8, 23 10.1186/1478-811X-8-23 [PMC free article] [PubMed] [Cross Ref]

Paulson A. F., Fang X., Ji H., Reynolds A. B., McCrea P. D. (1999). Misexpression of the catenin p120ctn1A perturbs Xenopus gastrulation but does not elicit Wnt-directed Axis specification. Dev. Biol. 207, 350-363 10.1006/dbio.1998.9158 [PubMed] [Cross Ref]

Piedra J., Miravet S., Castaño J., Pálmer H. G., Heisterkamp N., García de Herreros A., Duñach M. (2003). p120 Catenin-associated Fer and Fyn tyrosine kinases regulate beta-catenin Tyr-142 phosphorylation and beta-catenin-alpha-catenin Interaction. Mol. Cell. Biol. 23, 2287-2297 10.1128/MCB.23.7.2287-2297.2003 [PMC free article] [PubMed] [Cross Ref] 
Reya T., Clevers H. (2005). Wnt signalling in stem cells and cancer. Nature 434, 843-850 10.1038/nature03319 [PubMed] [Cross Ref]

Roura S., Miravet S., Piedra J., García de Herreros A., Duñach M. (1999). Regulation of E-cadherin/Catenin association by tyrosine phosphorylation. J. Biol. Chem. 274, 36734-36740 10.1074/jbc.274.51.36734 [PubMed] [Cross Ref]

Solanas G., Miravet S., Casagolda D., Castaño J., Raurell I., Corrionero A., de Herreros A. G., Duñach M. (2004). beta-Catenin and plakoglobin N- and C-tails determine ligand specificity. J. Biol. Chem. 279, 49849-49856 10.1074/jbc.M408685200 [PubMed] [Cross Ref]

Soto E., Yanagisawa M., Marlow L. A., Copland J. A., Perez E. A., Anastasiadis P. Z. (2008). p120 catenin induces opposing effects on tumor cell growth depending on E-cadherin expression. J. Cell Biol. 183, 737-749 10.1083/jcb.200805113 [PMC free article] [PubMed] [Cross Ref]

Vlad A., Röhrs S., Klein-Hitpass L., Müller O. (2008). The first five years of the Wnt targetome. Cell. Signal. 20, 795-802 10.1016/j.cellsig.2007.10.031 [PubMed] [Cross Ref]

Wu X., Tu X., Joeng K. S., Hilton M. J., Williams D. A., Long F. (2008). Rac1 activation controls nuclear localization of beta-catenin during canonical Wnt signaling. Cell 133, 340-353 10.1016/j.cell.2008.01.052 [PMC free article] [PubMed] [Cross Ref]

Yanagisawa M., Huveldt D., Kreinest P., Lohse C. M., Cheville J. C., Parker A. S., Copland J. A., Anastasiadis P. Z. (2008). A p120 catenin isoform switch affects Rho activity, induces tumor cell invasion, and predicts metastatic disease. J. Biol. Chem. 283, 18344-18354 10.1074/jbc.M801192200 [PMC free article] [PubMed] [Cross Ref]

Yu B., Martins I. R. S., Li P., Amarasinghe G. K., Umetani J., Fernandez-Zapico M. E., Billadeau D. D., Machius M., Tomchick D. R., Rosen M. K. (2010). Structural and energetic mechanisms of cooperative autoinhibition and activation of Vav1. Cell 140, 246-256 10.1016/j.cell.2009.12.033 [PMC free article] [PubMed] [Cross Ref]

Zhu G., Wang Y., Huang B., Liang J., Ding Y., Xu A., Wu W. (2012). A Rac1/PAK1 cascade controls $\beta$-catenin activation in colon cancer cells. Oncogene 31, 1001-1012 10.1038/onc.2011.294 [PubMed] [Cross Ref] 